\title{
Bleeding from major and minor duodenal papillae due to hemosuccus pancreaticus confirmed with side-view gastroduodenal endoscopy
}

\author{
Koichi Soga • Hideyuki Konishi • Takashi Miyajima • \\ Toshikazu Yoshikawa
}

Published online: 30 September 2011

(C) Indian Society of Gastroenterology 2011
A 46-year-old man was admitted to our hospital following hematemesis. Emergency direct-viewing gastroduodenoscopy revealed fresh bleeding from the major duodenal papilla. Further examination of both papillae by sideviewing endoscopy confirmed fresh bleeding from both the major and minor papillae (Fig. 1). Ultrasound and computed tomography $(\mathrm{CT})$ revealed a $20 \mathrm{~mm} \times 18 \mathrm{~mm}$ cystic mass in the body of the pancreas. The mass also demonstrated features consistent with chronic pancreatitis. Contrast enhanced CT showed that splenic artery pseudoaneurysm was communicating with the pseudocyst. A diagnosis of ruptured splenic artery aneurysm was made and angiography with embolization was successfully performed. Hemosuccus pancreaticus (HP) refers to hemorrhage arising from the major duodenal papilla via the pancreatic duct, is a rare cause of intermittent upper gastrointestinal bleeding. Difficulties in localizing the site of bleeding can sometimes delay treatment, which is especially significant in critically ill patients [1]. The characteristic feature of HP is intermittent bleeding from the papilla (major or minor) and the appearance of the papilla may be normal between the bleeding episodes. In case of high degree of suspicion, addition of antispasmodics and repeated endoscopy with drugs to relax the papillary sphincter if necessary, has been recommended.

\section{K. Soga $(\bowtie)$}

Department of Internal Medicine, Nishijin Hospital,

1035 Mizomae-cho, Kamigyo-ku,

Kyoto 602-8319, Japan

e-mail:sogatti@koto.kpu-m.ac.jp

H. Konishi $\cdot$ T. Yoshikawa

Department of Molecular Gastroenterology and Hepatology,

Kyoto Prefectural University of Medicine,

Kyoto, Japan

\section{K. Soga $\cdot$ T. Miyajima}

Department of Gastroenterology, Uji Takeda Hospital,

Kyoto, Japan
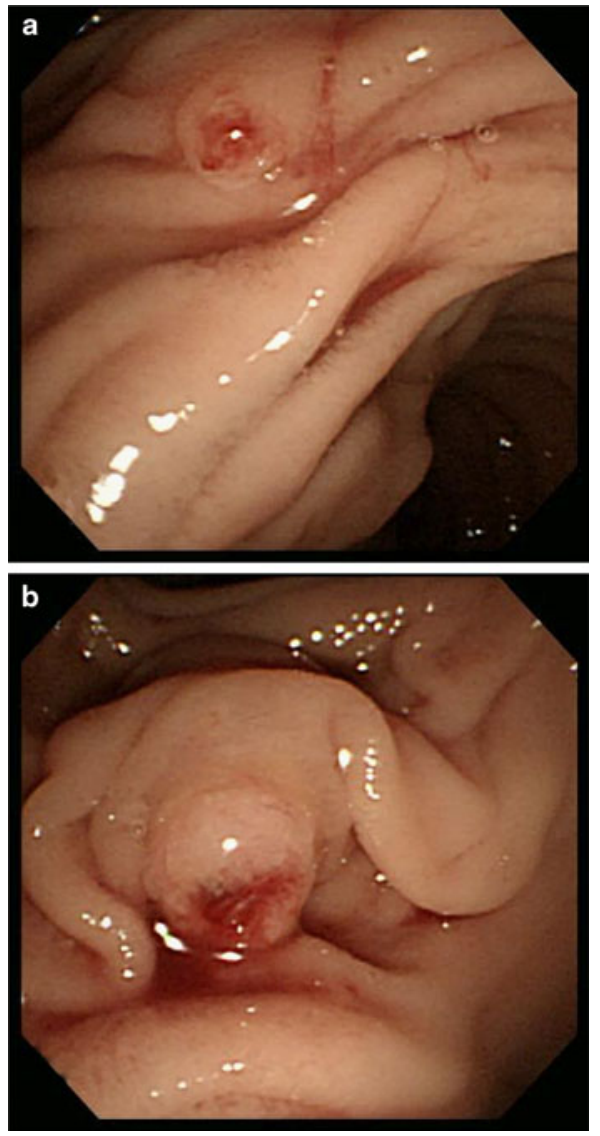

Fig. 1 Side-viewing gastroduodenoscopy of hemosuccus pancreaticus: a View of the minor duodenal papilla, b View of the major duodenal papilla

\section{Reference}

1. Massani M, Bridda A, Caratozzolo E, Bonariol L, Antoniutti M, Bassi N. Hemosuccus pancreaticus due to primary splenic artery aneurysm: a diagnostic and therapeutic challenge. JOP. 2009;10:48-52. 